\title{
Refractive index of a transparent liquid measured with a concave mirror
}

\author{
Amitabh Joshi ${ }^{1}$ and Juan D Serna ${ }^{2}$ \\ ${ }^{1}$ Department of Physics, Eastern Illinois University, Charleston, IL 61920, USA \\ ${ }^{2}$ School of Mathematical and Natural Sciences, University of Arkansas at Monticello, \\ Monticello, AR 71656, USA \\ E-mail: ajoshi@eiu.edu, serna@uamont.edu
}

\begin{abstract}
This paper describes the spherical concave mirror method for measuring the index of refraction of transparent liquids. We derived the refractive index equation using Snell's law and the small-angle approximation. We also verified the validity of this method using the traditional spherical mirror and thin-lens Gaussian equations.

PACS numbers: 01.50.Pa, 42.15.-i, 01.30.1b
\end{abstract}

\section{Introduction}

Measuring the refractive index $n$ of a substance or medium is part of every introductory physics lab. Various approaches to determine this index have been developed over the years based on the different ways light reflects and transmits in the medium.

With the introduction of lasers in basic physics courses, a number of these methods have become accessible to the undergraduate physics laboratory. Several of these techniques are highly accurate and use specific optical equipment, like spectrometers, interferometers, or microscopes. In the laboratory, the method instructors choose to measure the refractive index of a liquid depends on different factors, such as the optical properties of the substance they want to study, or simply the resources available at hand, that in many cases, are very limited.

Among all those different methods, the spherical concave mirror filled with a liquid makes itself an excellent alternative to measure the refractive index of the liquid, specially when no fancy apparatuses are available, and the accuracy of the measurements is not critical.

In this paper, we would like to present a simple geometrical derivation of the refractive index of a transparent liquid that is obtained using a spherical concave mirror. This derivation relies mostly on Snell's law and the small-angle approximation. In addition, we also use Gaussian optics equations for mirrors and thin-lenses to verify the validity of the refractive index equation. 
This method is based on the measurements of the actual and apparent position of the centre of curvature of the mirror, when it is empty and filled with a liquid, respectively. The laws of reflection and refraction are essential to understand the physics behind this method. Students measuring indices of refraction of liquids using this technique are introduced gradually with the concepts of reflection and refraction of light, Snell's law, image formation by spherical mirrors, and Gaussian optics.

\section{Spherical mirrors}

Let us first consider the spherical concave mirror shown in figure 1. The optical axis is the radial line through the centre of the mirror that intersects its surface at the vertex point $V$. Some relevant points on the optical axis are the centre of curvature $C$, and the focal point $F$. The centre of curvature coincides with the centre of the sphere of which the mirror forms a section. At the focal point, rays parallel to the optical axis and incident on the concave mirror, intersect after being reflected by the mirror's surface [1]. The distance $C V$ between the centre of curvature and the vertex is equal to the radius of

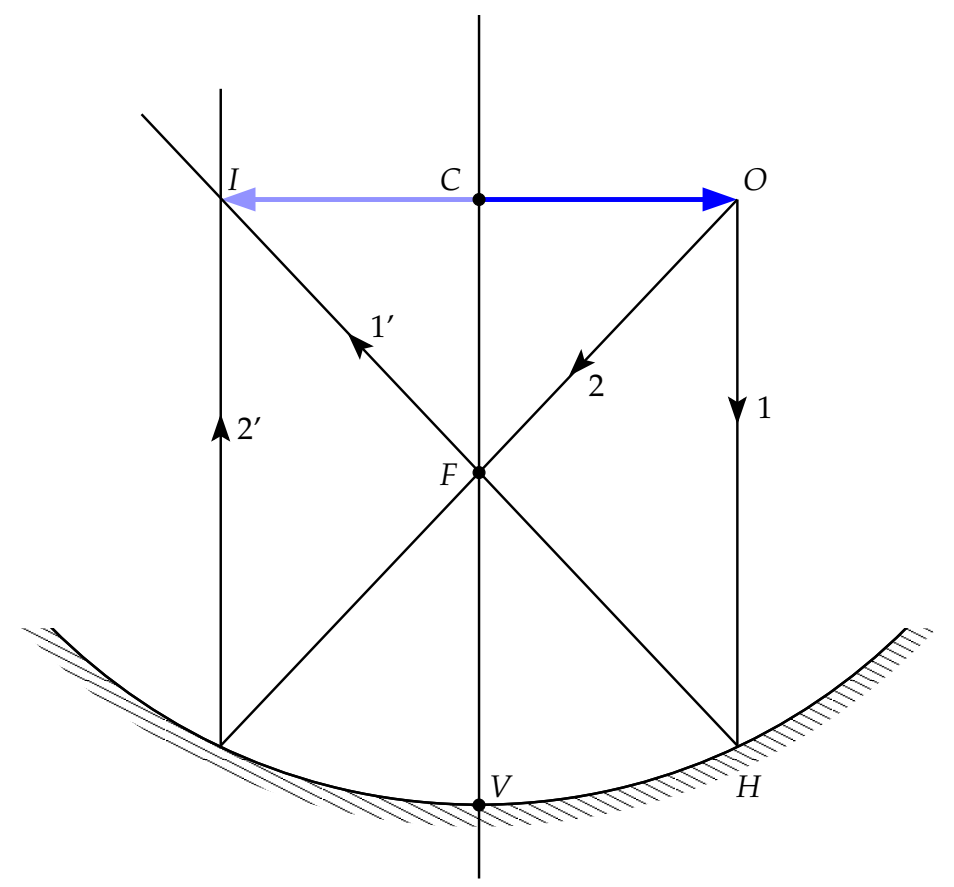

Figure 1. Positions of the focal point and centre of curvature of a spherical concave mirror. Rays 1 and 2 , and their respective reflections $1^{\prime}$ and $2^{\prime}$ in the mirror, determine the location of the image.

the sphere and is called the radius of curvature $R$. Similarly, the distance $F V$ between the focal point of the mirror and the vertex is known as the focal length $f$.

In general, when the rays are close to the optical axis - that is, for the small-angle 
approximation, the focal length can be shown to be half of the radius of curvature [2]:

$$
f=\frac{R}{2} \text {. }
$$

The location and nature of the image formed by a spherical mirror can be determined by graphical ray-tracing techniques [3]. To find the conjugate image point $I$ of an object point $O$ located at the centre of curvature $C$, the paths of any two rays leaving $O$ are sufficient [4]. We first use the so-called parallel ray 1 that is incident along a path parallel to the optical axis, strikes the mirror at point $H$, and is reflected through the focal point $F$ as ray $1^{\prime}$. Next, we use the so-called focal ray 2 that passes through the focal point and is reflected parallel to the optical axis as ray $2^{\prime}$. The image point $I$ is formed where the two rays $1^{\prime}$ and $2^{\prime}$ intersect. This image is real, inverted, located at the centre of curvature $C$, and has the same size of the object, as shown in figure 1.

\section{The experiment}

Measuring the refractive index $n$ of a transparent liquid, like water, using a spherical concave mirror is based on locating the actual and apparent centres of curvature of the mirror, when it is empty and filled with a thin layer of water, respectively.

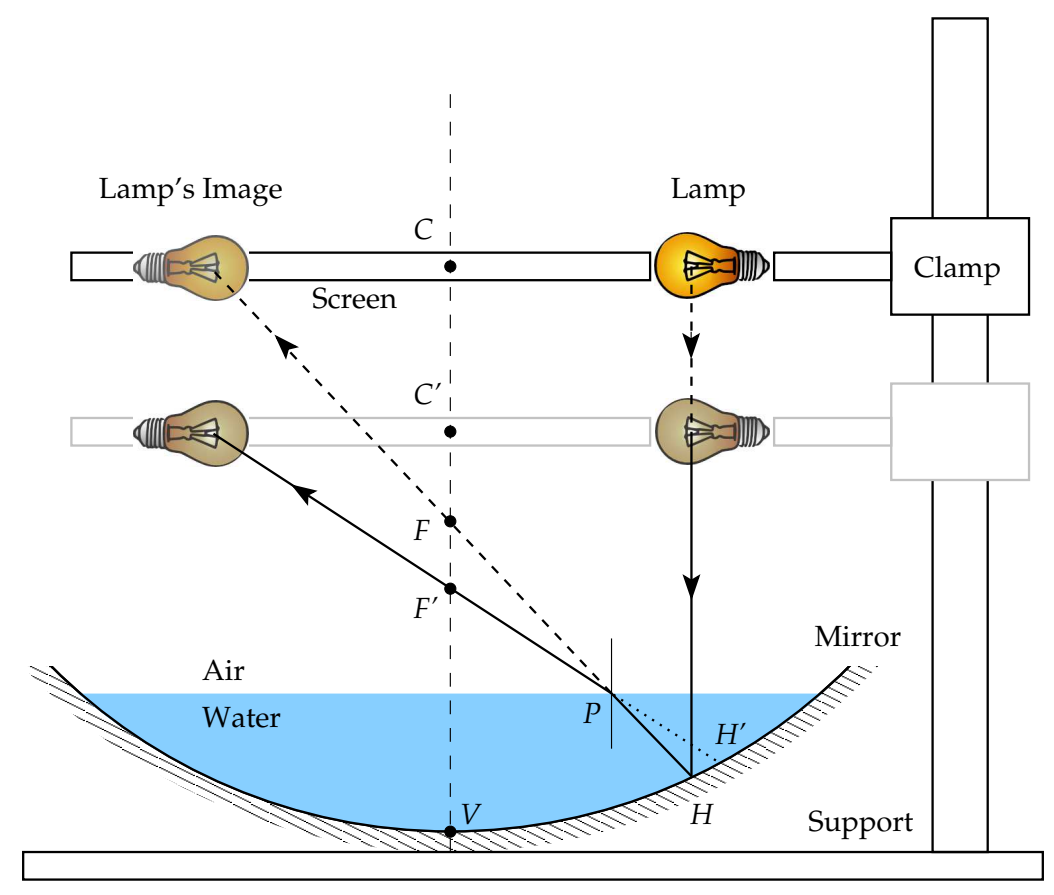

Figure 2. Schematics of the spherical concave mirror experiment for measuring the refractive index of water. The support holding the lamp is moved up and down until a sharp image of the lamp is formed on the screen.

The typical experimental setup (see figure 2) consists on a mirror, a support with a vertical rod, and a clamp holding a lamp (source of light) and a screen horizontally [5]. When there is no water in the mirror, the centre of curvature is located at $C$, and the 
radius of curvature is $R=C V$. From (1), the focal length of the concave mirror is $f=F V=R / 2$. When the mirror is filled with water, the apparent centre of curvature $C^{\prime}$ moves down, and the apparent radius of curvature becomes $R^{\prime}=C^{\prime} V$. The new focal length is given by $f^{\prime}=F^{\prime} V=R^{\prime} / 2$.

To obtain the refractive index of water $n_{\mathrm{w}}$, the clamp that holds the lamp and the screen are moved to position $C$ until a sharp image of the lamp is formed by the empty mirror. The radius of curvature $R$ is then measured. Next, the mirror is filled with a thin layer of water, and the lamp and screen moved down until a new sharp image of the lamp is formed on the screen. This position corresponds to the apparent centre of curvature $C^{\prime}$. The new radius of curvature $R^{\prime}$ is then measured. The refractive index can be obtained by using the equation

$$
n_{\mathrm{w}}=\frac{R}{R^{\prime}} \text {. }
$$

\section{The Snell's law approach}

When the point object $O$ is placed at the centre of curvature $C$, and the mirror is empty (no water has been poured in), we may use the same graphical ray-tracing methods of figure 1 to locate the conjugate image point $I$. Ray $1_{\mathrm{a}}$ leaves point $O$ parallel to the optical axis, strikes the mirror at point $H$ and reflects as ray $1_{a}^{\prime}$. This ray intersects the principal axis at focal point $F$. The focal length is then $f=F V$. The conjugate image point $I$ is formed at the centre of curvature $C$, as illustrated in figure 3. The image is real, inverted and has the same size of the object.

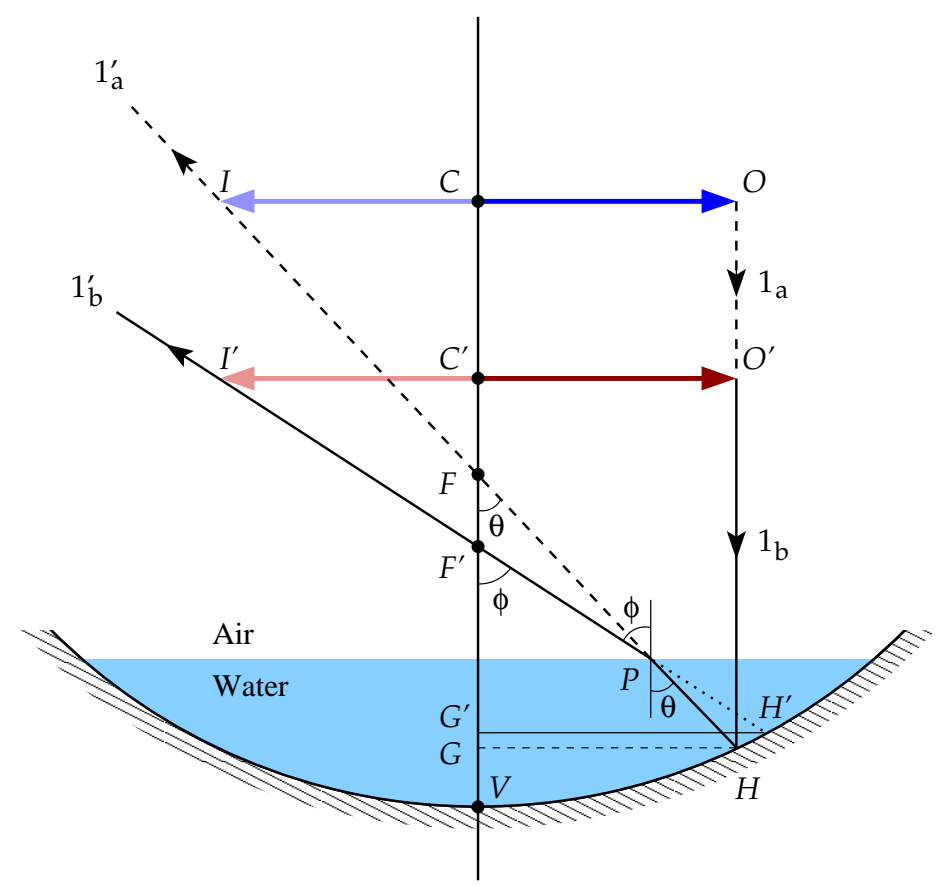

Figure 3. Formation of the images at the positions of the actual and apparent centres of curvature. 
Now, when the mirror is filled with a thin layer of water, the magnitude of its focal length decreases, and the apparent centre of curvature $C^{\prime}$ gets closer to the vertex $V$ of the mirror. Ray $1_{\mathrm{a}}$ leaves object point $O$ parallel to the optical axis, strikes the mirror at point $H$ and then reflects. The reflected ray refracts from water into air bending away from the surface normal at the point of incidence $P$, for the refractive index of water $n_{\mathrm{w}}$ is bigger than that of air $n_{\mathrm{a}}$. The refracted ray $1_{\mathrm{b}}^{\prime}$ intersects the optical axis at the new focal point $F^{\prime}$. The new focal length becomes $f^{\prime}=F^{\prime} V$. If the object is placed at the apparent centre of curvature $C^{\prime}$, and labelled object point $O^{\prime}$, the conjugate image point $I^{\prime}$ is found at the apparent centre of curvature $C^{\prime}$. The image is real, inverted, and has the same size of the object (see figure 3).

Using Snell's law at surface point $P$, we get

$$
n_{\mathrm{w}} \sin \theta=n_{\mathrm{a}} \sin \phi,
$$

where $n_{\mathrm{w}}$ and $n_{\mathrm{a}}$ are the refractive indices of water and air, respectively. From this point on, we will assume $n_{\mathrm{a}} \approx 1.00$.

Figure 3 shows that from triangles $F H G$ and $F^{\prime} H^{\prime} G^{\prime}$

$$
\begin{aligned}
& H G=F H \sin \theta \\
& H^{\prime} G^{\prime}=F^{\prime} H^{\prime} \sin \phi .
\end{aligned}
$$

The backward extension of the refracted ray $1_{\mathrm{b}}^{\prime}$ strikes the mirror at point $H^{\prime}$. If we assume that the focal lengths $F$ and $F^{\prime}$ are large compared to the thickness of the water layer in the mirror, points $G$ and $G^{\prime}$, as well as, $H$ and $H^{\prime}$ are very close to each other. Therefore, we can make the following approximations for the geometrical lengths:

$$
\begin{aligned}
& H^{\prime} G^{\prime} \approx H G \\
& F^{\prime} H^{\prime} \approx F^{\prime} H .
\end{aligned}
$$

Physically, points $H$ and $H^{\prime}$, as well as $G$ and $G^{\prime}$ are not coinciding but are located nearby. Using (5) in (4b), we may write

$$
H G=F^{\prime} H \sin \phi .
$$

Since the left hand sides of $(4 a)$ and $(4 b)$ are the same, we may equate both equations to obtain

$$
F H \sin \theta=F^{\prime} H \sin \phi .
$$

Using (3) in (7), we obtain

$$
\frac{F H}{F^{\prime} H}=\frac{\sin \phi}{\sin \theta}=n_{\mathrm{w}} .
$$

If the angles $\theta$ and $\phi$ are small, then this angles can be replaced by their tangents (small-angle approximation). Furthermore, the distance $G V$ in figure 3 , the sagittal depth of the surface [6], is also small, and we may neglect it. Therefore, we can write

$$
n_{\mathrm{w}}=\frac{\tan \phi}{\tan \theta}=\frac{f}{f^{\prime}} .
$$


Finally, using (11), we can express the refractive index of water in terms of the actual and apparent radii of curvature as follows

$$
n_{\mathrm{w}}=\frac{R}{R^{\prime}}
$$

\section{The silvered lens analogue}

An expression for the refractive index of the liquid may be obtained also from the spherical mirror and thin-lens formulas

$$
\begin{aligned}
& \frac{1}{s}+\frac{1}{s^{\prime}}=-\frac{2}{r} \\
& \frac{n_{1}}{s}+\frac{n_{2}}{s^{\prime}}=\frac{n_{2}-n_{1}}{r},
\end{aligned}
$$

where $s$ is the object distance, $s^{\prime}$ is the image distance, and $r$ is the radius of curvature; $n_{1}$ and $n_{2}$ are the refractive indices of glass (liquid) and air, respectively.

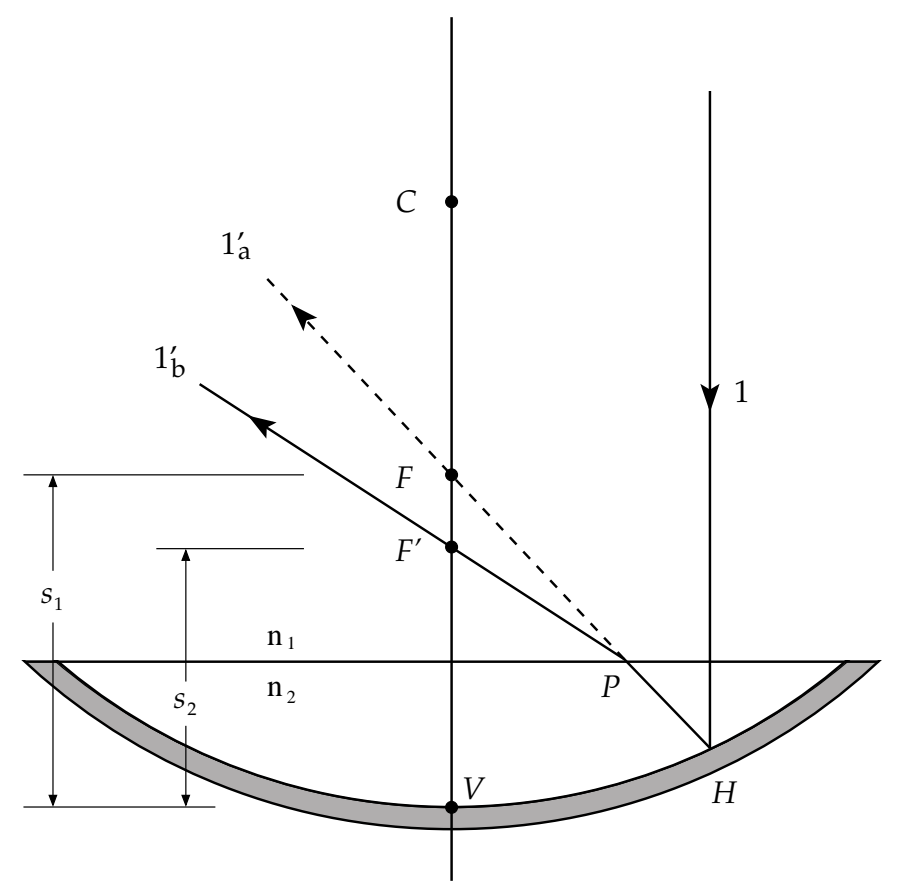

Figure 4. Schematic of the silvered lens analogue.

Consider a thin, plano-convex lens of radius of curvature $R=C V$ and silvered on its curved side, as illustrated in figure 4 . For an object at infinity $(s \rightarrow \infty)$, incident rays are parallel to the optical axis. After reflection at the curved face, we may use (11a) to get

$$
\frac{1}{\infty}+\frac{1}{s_{1}}=-\frac{2}{R}
$$

Thus, $s_{1}=-R / 2=f$. 
Now, after refraction at the plane face $(r \rightarrow \infty)$, and using (11b) with $s=s_{1}$, $s^{\prime}=s_{2}, n_{1}=n_{\mathrm{w}}$, and $n_{2}=1$, we obtain

$$
\frac{n_{\mathrm{w}}}{-R / 2}+\frac{1}{s_{2}}=0
$$

Then, $s_{2}=R / 2 n=f^{\prime}$ (see figure 4).

Finally, taking the ratio of the two focal lengths, we get

$$
\frac{f}{f^{\prime}}=\frac{R / 2}{R / 2 n_{\mathrm{w}}}=n_{\mathrm{w}}
$$

which is in accordance with (9) (see reference [3]).

\section{Conclusions}

The experiment described in this paper gives a simple and effective method of measuring the refractive index of water (or any transparent liquid) using a spherical concave mirror.

A valuable pedagogical consideration of this method is that, students may observe that a real image of the object is formed at the centre of curvature of the empty mirror when the object is located at the same point. Similarly, a real image is formed at the apparent centre of curvature when the mirror is filled with water, if the object is located at the same place. This provides an easy way of finding the refractive index of the liquid by measuring the ratio between the actual and apparent radii of curvature. In addition, by using Snell's law and reflection of rays in a concave spherical mirror, they should notice that the images are not only real, but inverted, and have the same size of the object.

As shown by the Snell's law approach, with the concave mirror method we can get an expression for the refractive index of a liquid using merely Snell's law and the small angle approximation (paraxial approximation), and avoiding complications introduced by the thin-lens equation. However, in the silvered mirror analogue, we use the spherical mirror and thin-lens equations to verify the validity of the refractive index equation obtained using the Snell's law.

This experiment is suitable for the undergraduate physics laboratory. It is easy to setup and perform. It has been carried out successfully in the lab providing accurate numerical values of the refractive index of water and other transparent liquids.

\section{Acknowledgments}

Author (AJ) gratefully acknowledges funding support from RCSA.

\section{References}

[1] Wilson J D, Buffa A J and Lou B 2010 College physics, 7th ed. (San Francisco: Addison-Wesley) p 782

[2] Hecht E 2002 Optics, 4th ed. (Reading: Addison-Wesley) p 183 
[3] Pedrotti F L, Pedrotti L M and Pedrotti L S 2007 Introduction to optics, 3th ed. (Upper Saddle River: Pearson/Prentice Hall) p 31, 49

[4] Suppapittayaporn D, Panijpan B and Emarat N 2010 Can We Trace Arbitrary Rays To Locate an Image Formed by a Thin Lens? Phys. Teach. 48 256-257

[5] Rachna S. 2009 Physics Lab Manual-XII (Together With), 2nd ed. (New Delhi: Rachna Sagar Pvt. Ltd.) p 86

[6] Blaker J W and Rosenblum W M 1993 Optics-An Introduction for Students of Engineering (New York: Macmillan Pub. Co.) p 15 